\title{
Two Causal Principles for Improving Visual Dialog
}

\author{
Jiaxin Qi ${ }^{* 1} \quad$ Yulei Niu $^{* 2}$ Jianqiang Huang ${ }^{1,3}$ Hanwang Zhang ${ }^{1}$ \\ ${ }^{1}$ Nanyang Technological University, ${ }^{2}$ Renmin University of China, \\ ${ }^{3}$ Damo Academy, Alibaba Group, \\ jiaxin003@e.ntu.edu.sg, niu@ruc.edu.cn, jianqiang.jqh@gmail.com, hanwangzhang@ntu.edu.sg
}

\begin{abstract}
This paper unravels the design tricks adopted by us - the champion team MReaL-BDAI - for Visual Dialog Challenge 2019: two causal principles for improving Visual Dialog (VisDial). By "improving", we mean that they can promote almost every existing VisDial model to the stateof-the-art performance on the leader-board. Such a major improvement is only due to our careful inspection on the causality behind the model and data, finding that the community has overlooked two causalities in VisDial. Intuitively, Principle 1 suggests: we should remove the direct input of the dialog history to the answer model, otherwise a harmful shortcut bias will be introduced; Principle 2 says: there is an unobserved confounder for history, question, and answer, leading to spurious correlations from training data. In particular, to remove the confounder suggested in Principle 2, we propose several causal intervention algorithms, which make the training fundamentally different from the traditional likelihood estimation. Note that the two principles are model-agnostic, so they are applicable in any VisDial model. The code is available at https://github. $\mathrm{com} / \mathrm{simpleshinobu/visdial-principles.}$
\end{abstract}

\section{Introduction}

Given an image $I$, a dialog history of past Q\&A pairs: $H=\left\{\left(Q_{1}, A_{1}\right), \ldots,\left(Q_{t-1}, A_{t-1}\right)\right\}$, and the current $t$-th round question $Q$, a Visual Dialog (VisDial) agent [9] is expected to provide a good answer $A$. Our community has always considered VQA [5] and VisDial as sister tasks due to their similar settings: Q\&A grounded by $I$ (VQA) and Q\&A grounded by $(I, H)$ (VisDial). Indeed, from a technical point view - just like the VQA models - a typical VisDial model first uses encoder to represent $I, H$, and $Q$ as vectors, and then feed them into decoder for answer $A$. Thanks to the recent advances in encoder-decoder frameworks in VQA $[22,38]$ and natural language process-

\footnotetext{
*Equal contribution
}

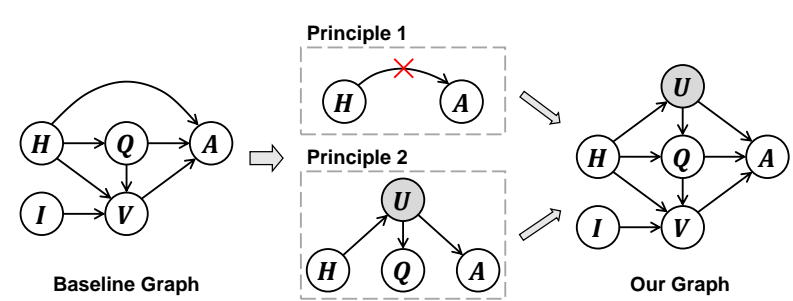

Figure 1. Causal graphs of VisDial models (baseline and ours). $H$ : dialog history. $I$ : image. $Q$ : question. $V$ : visual knowledge. $A$ : answer. $U$ : user preference. Shaded $U$ denotes unobserved confounder. See Section 3.2 for detailed definitions.

ing [39], the performance (NDCG [1]) of VisDial in literature is significantly improved from the baseline 51.63\% [2] to the state-of-the-art $64.47 \%$ [11].

However, in this paper, we want to highlight an important fact: VisDial is essentially NOT VQA with history! And this fact is so profound that all the common heuristics in the vision-language community - such as the multimodal fusion $[38,46]$ and attention variants $[22,25,26]$ - cannot appreciate the difference. Instead, we introduce the use of causal inference $[27,28]$ : a graphical framework that stands in the cause-effect interpretation of the data, but not merely the statistical association of them. Before we delve into the details, we would like to present the main contributions: two causal principles, rooted from the analysis of the difference between VisDial and VQA, which lead to a performance leap - a farewell to the $60 \%$-s and an embrace for the $70 \%$-s - for all the baseline VisDial models ${ }^{1}$ in literature $[9,21,41,26]$, promoting them to the state-of-the-art in Visual Dialog Challenge 2019 [2].

Principle 1 (P1): Delete link $H \rightarrow A$.

Principle 2 (P2): Add one new (unobserved) node $U$ and three new links: $U \leftarrow H, U \rightarrow Q$, and $U \rightarrow A$.

Figure 1 compares the causal graphs of existing VisDial models and the one applied with the proposed two principles. Although a formal introduction of them is given in

\footnotetext{
${ }^{1}$ Only those with codes\&reproducible results due to resource limit.
} 


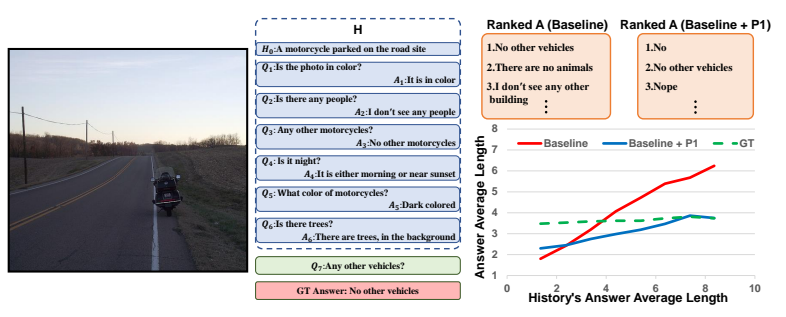

(a) A Typical $H \rightarrow A$ Bias

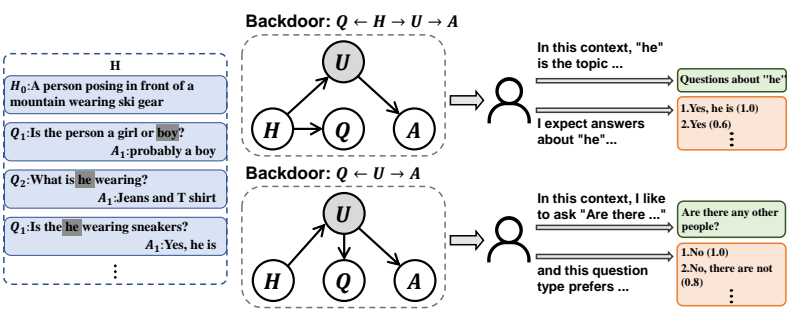

(b) User Preference

Figure 2. The illustrative motivations of the two causal principles: (a) P1 and (b) P2.

Section 3.2, now you can simply understand the nodes as data types and the directed links as modal transformations. For example, $V \rightarrow A$ and $Q \rightarrow A$ indicate that answer $A$ is the effect caused by visual knowledge $V$ and question $Q$, through a transformation, e.g., a multi-modal encoder.

P1 suggests that we should remove the direct input of dialog history to the answer model. This principle contradicts most of the prevailing VisDial models [9, 15, 41, 26, 43, 16, 11, 32], which are based on the widely accepted intuition: the more features you input, the more effective the model is. It is mostly correct, but only with our discretion of the data generation process. In fact, the VisDial [9] annotators were not allowed to copy from the previous Q\&A, i.e., $H \nrightarrow A$, but were encouraged to ask consecutive questions including co-referenced pronouns like "it" and "those", i.e., $H \rightarrow Q$, and thus the answer $A$ is expected to be only based on question $Q$ and reasoned visual knowledge $V$. Therefore, a good model should reason over the context $(I, H)$ with $Q$ but not to memorize the bias. However, the direct path $H \rightarrow A$ will contaminate the expected causality. Figure 2(a) shows a very ridiculous bias observed in all baselines without $\mathrm{P}$ : the top answers are those with length closer to the average length in the history answers. We will offer more justifications for P1 in Section 4.1.

P2 implies that the model training only based on the association between $(I, H, Q)$ and $A$ is spurious. By "spurious", we mean that the effect on $A$ caused by $(I, H, Q)$ - the goal of VisDial - is confounded by an unobserved variable $U$, because it appears in every undesired causal path (a.k.a., backdoor [28]), which is an indirect causal link from input $(I, H, Q)$ to output $A: Q \leftarrow U \rightarrow A$ and $Q \leftarrow H \rightarrow U \rightarrow A$. We believe that such unob- served $U$ should be users as the VisDial dataset essentially brings humans in the loop. Figure 2(b) illustrates how the user's hidden preference confounds them. Therefore, during training, if we focus only on the conventional likelihood $P(A \mid I, H, Q)$, the model will inevitably be biased towards the spurious causality, e.g., it may score answer "Yes, he is" higher than "Yes", merely because the users prefer to see a "he" appeared in the answer, given the history context of "he". It is worth noting that the confounder $U$ is more impactful in VisDial than in VQA, because the former encourages the user to rank similar answers subjectively while the latter is more objective. A plausible explanation might be: VisDial is interactive in nature and a not quite correct answer is tolerable in one iteration (i.e., dense prediction); while VQA has only one chance, which demands accuracy (i.e., one-hot prediction).

By applying P1 and P2 to the baseline causal graph, we have the proposed one (the right one in Figure 1), which serves as a model-agnostic roadmap for the causal inference of VisDial. To remove the spurious effect caused by $U$, we use the do-calculus [28] $P(A \mid d o(I, H, Q))$, which is fundamentally different from the conventional likelihood $P(A \mid I, H, Q)$ : the former is an active intervention, which cuts off $U \rightarrow Q$ and $H \rightarrow Q$, and sample (where the name "calculus" is from) every possible $U \mid H$, seeking the true effect on $A$ only caused by $(I, H, Q)$; while the latter likelihood is a passive observation that is affected by the existence of $U$. The formal introduction and details will be given in Section 4.3. In particular, given the fact that once the dataset is ready, $U$ is no longer observed, we propose a series of effective approximations in Section 5.

We validate the effectiveness of $\mathrm{P} 1$ and $\mathrm{P} 2$ on the most recent VisDial v1.0 dataset. We show significant boosts (absolute NDCG) by applying them in 4 representative baseline models: LF [9] ( $\uparrow 16.42 \%)$, HCIAE [21] ( $\uparrow 15.01 \%)$, CoAtt [41] ( $\uparrow 15.41 \%)$, and RvA [26] ( $\uparrow 16.14 \%)$. Impressively, on the official test-std server, we use an ensemble model of the most simple baseline LF [9] to beat our 2019 winning performance by $0.2 \%$, a more complex ensemble to beat it by $0.9 \%$, and lead all the single-model baselines to the state-of-the-art performances.

\section{Related Work}

Visual Dialog. Visual Dialog $[9,10]$ is more interactive and challenging than most of the vision-language tasks, e.g., image captioning [45, 44, 4] and VQA [5, 38, 37, 36]. Specifically, Das et al. [9] collected a large-scale free-form visual dialog dataset VisDial [7]. They applied a novel protocol: during the live chat, the questioner cannot see the picture and asks open-ended questions, while the answerer gives free-form answers. Another dataset GuessWhat?! proposed by [10] is a goal-driven visual dialog: questioner should locate an unknown object in a rich image scene by asking a 
sequence of closed-ended "yes/no" questions. We apply the first setting in this paper. Thus, the key difference is that the users played an important role in the data collection process.

All of the existing approaches in the VisDial task are based on the typical encoder-decoder framework [15, 12, $33,11,32,47]$. They can be categorized by the usage of history. 1) Holistic: they treat history as a whole to feed into models like HACAN [43], DAN [16] and CorefNMN [18]. 2) Hierarchical: they use a hierarchical structure to deal with history like HRE [9]. 3) Recursive: RvA [26] uses a recursive method to process history. However, they all overlook the fact that the history information should not be directly fed to the answer model (i.e., our proposed Principle 1). The baselines we used in this paper are LF [9]: the earliest model, HCIAE [21]: the first model to use history hierarchical attention, CoAtt [41]: the first one to a co-attention mechanism, and RvA [26]: the first one for a tree-structured attention mechanism.

Causal Inference. Recently, some works [24, 6, 23, 34, 40] introduced causal inference into machine learning, trying to endow models the abilities of pursuing the cause-effect. In particular, we use the Pearl's structural causal model (SCM) proposed by [28] to hypothesize the data generation process, which is a model-agnostic framework that reflects the nature of the data.

\section{Visual Dialog in Causal Graph}

In this section, we formally introduce the visual dialog task and describe how the popular encoder-decoder framework follows the baseline causal graph shown in Figure 1. More details of causal graph can be found in [28, 29].

\subsection{Visual Dialog Settings}

Settings. According to the definition of VisDial task proposed by Das et al. [9], at each time $t$, given input image $I$, current question $Q_{t}$, dialog history $H=$ $\left\{C,\left(Q_{1}, A_{1}\right), \ldots,\left(Q_{t-1}, A_{t-1}\right)\right\}$, where $C$ is the image caption, $\left(Q_{i}, A_{i}\right)$ is the $i$-th round Q\&A pair, and a list of 100 candidate answers $A_{t}=\left\{A_{t}^{(1)}, \ldots, A_{t}^{(100)}\right\}$. A VisDial model is evaluated by ranking candidate answers $A_{t}$.

Evaluation. Recently, the ranking metric Normalized Discounted Cumulative Gain (NDCG) is adopted by the VisDial community [1]. It is different from the classification metric (e.g., top-1 accuracy) used in VQA. It is more compatible with the relevance scores of the answer candidates in VisDial rated by humans. NDCG requires to rank relevant candidates in higher places, rather than just to select the ground-truth answer.

\subsection{Encoder-Decoder as Causal Graph}

We first give the definition of causal graph, then revisit the encoder-decoder framework in existing methods using the elements from the baseline graph in Figure 1.
Causal Graph. Causal graph [28], as shown in Figure 1, describes how variables interact with each other, expressed by a directed acyclic graph $\mathcal{G}=\{\mathcal{N}, \mathcal{E}\}$ consisting of nodes $\mathcal{N}$ and directed edges $\mathcal{E}$ (i.e., arrows). $\mathcal{N}$ denote variables, and $\mathcal{E}$ (arrows) denote the causal relationships between two nodes, i.e., $A \rightarrow B$ denotes that $A$ is the cause and $B$ is the effect, meaning the outcome of $B$ is caused by $A$. Causal graph is a highly general roadmap specifying the causal dependencies among variables.

As we will discuss in the following part, all of the existing methods can be revisited in the view of the baseline graph shown in Figure 1.

Feature Representation and Attention in Encoder. Visual feature is denoted as node $I$ in the baseline graph, which is usually a fixed feature extracted by FasterRCNN [31] based on ResNet backbone [13] pre-trained on Visual Genome [19]. For language feature, the encoder firstly embeds sentence into word vectors, followed by passing the RNN $[14,8]$ to generate features of question and history, which are denoted as $\{Q, H\}$.

Most of existing methods apply attention mechanism [42] in encoder-decoder to explore the latent weights for a set of features. A basic attention operation can be represented as $\tilde{\boldsymbol{x}}=\operatorname{Att}(\mathcal{X}, \mathcal{K})$ where $\mathcal{X}$ is the set of features need to attend, $\mathcal{K}$ is the key (i.e., guidance) and $\tilde{\boldsymbol{x}}$ is the attended feature of $\mathcal{X}$. Details can be found in most visual dialog methods $[21,41,43]$. In the baseline graph, the subgraph $\{I \rightarrow V, Q \rightarrow V, H \rightarrow Q \rightarrow V\}$ denotes a series of attention operations for visual knowledge $V$. Note that the implementation of the arrows are not necessarily independent, such as co-attention [41], and the process can be written as Input : $\{I, Q, H\} \Rightarrow$ Output : $\{V\}$, where possible intermediate variables can be added as mediator nodes into the original arrows. However, without loss of generality, these mediators do not affect the causalities in the graph.

Response Generation in Decoder. After obtaining the features from the encoder, existing methods will fuse them and feed the fused ones into a decoder to generate an answer. In the baseline graph, node $A$ denotes the answer model that decodes the fused features from $\{H \rightarrow A, Q \rightarrow A, V \rightarrow A\}$ and then transforms them into an answer sentence. In particular, the decoder can be generative, i.e., to generate an answer sentence using RNN; or discriminative, i.e., to select an answer sentence by using candidate answer classifiers.

\section{Two Causal Principles}

\subsection{Principle 1}

When should we draw an arrow from one node pointing to another? According to the definition in Section 3.2, the criterion is that if the node is the cause and the other one is the effect. Intrigued, let's understand P1 by discussing the rationale behind the "double-blind" review pol- 
icy. Given three variables: "Well-known Researcher" $(R)$, "High-quality Paper" $(P)$, and "Accept" $(A)$. From our community common sense, we know that $R \rightarrow P$ since top researchers usually lead high-quality research, and $P \rightarrow A$ is even more obvious. Therefore, for the good of the community, the double-blind prohibits the direct link $R \rightarrow A$ by author anonymity, otherwise the bias such as personal emotions and politics from $R$ may affect the outcome of $A$.

The story is similar in VisDial. Without loss of generality, we only analyze the path $H \rightarrow Q \rightarrow A$. If we inspect the role of $H$, we can find that it is to help $Q$ resolve some co-references like "it" and "their". As a result, $Q$ listens to $H$. Then, we use $Q$ to obtain $A$. Here, $Q$ becomes a mediator which cuts off the direct association between $H$ and $A$ that makes $P(A \mid Q, H)=P(A \mid Q)$, like the "High-quality Paper" that we mentioned in the previous story. However, if we set an arrow from $H$ to $A: H \rightarrow A$, the undesirable bias of $H$ will be learned for the prediction of $A$, that hampers the natural process of VisDial, such as the interesting bias illustrated in Figure 2(a). Another example is discussed in Figure 4 that $A$ prefers to match the words in $H$, even though they are literally nonsense about $Q$ if we add the direct link $H \rightarrow A$. After we apply P1, these phenomena will be relieved, such as the blue line illustrated in Figure 2(a), which is closer to the NDCG ground truth average answer length, denoted as the green dashed line. Please refer to other qualitative studies in Section 6.5.

\subsection{Principle 2}

Before discussing P2, we first introduce an important concept in causal inference [28]. In causal graph, the forklike pattern in Figure 3(a) contains a confounder $U$, which is the common cause for $Q$ and $A$ (i.e., $Q \leftarrow U \rightarrow A$ ). The confounder $U$ opens a backdoor path started from $Q$, making $Q$ and $A$ spuriously correlated even if there is no direct causality between them.

In the data generation process of VisDial, we know that not only both of the questioner and answerer can see the dialog history, but also the answer annotators can look at the history when annotating the answer. Their preference after seeing the history can be understood as a part of the human nature or subtleties conditional on a dialog context, and thus it has a causal effect on both $Q$ and $A$. Moreover, due to the fact that the preference is nuanced and uncontrollable, we consider it as an unobserved confounder for $Q$ and $A$.

It is worth noting that the confounder hinders us to find the true causal effect. Let's take the graph in Figure 3(b) as an example. The causal effect from $Q$ to $A$ is 0 ; however, we can quickly see that $P(A \mid Q)-P(A)$ is nonzero because $Q$ and $A$ are both influenced by $U$ and thus are correlated (thanks to Reichenbach's common cause principle [28]). That is, if we are given $Q$, the any likelihood change for $A$ will be sensible compared to nothing is given.

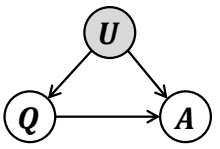

(a) Confounder $U$

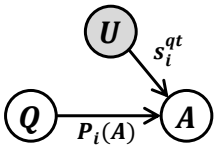

(d) Question Type

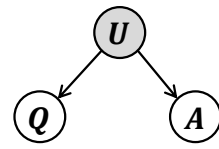

(b) Spurious Relation

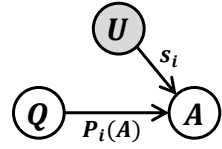

(e) Score Sampling

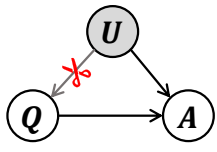

(c) do-operator

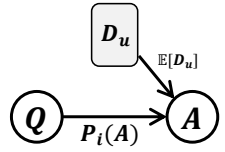

(f) Hidden Dictionary
Figure 3. Example of confounder, do-operator and sketch causal graphs of our three attempts to de-confounder

Therefore, if we consider $P(A \mid Q)$ as our VisDial model, it will still predict nonsense answers even if $Q$ has nothing to do with $A$. As illustrated in Figure 2(b), model will prefer the candidates about "he" even though $Q$ is not given, that means it captures the confounder $U$ but not the true rationale between $Q$ and $A$. Next, we will introduce a powerful technique that makes the $Q$ and $A$ in Figure 3(b) "independent", i.e., no causal relation.

\section{3. $d o$-calculus.}

The technique is do-calculus introduced in $[28,29]$. Specifically, $\operatorname{do}(Q=q)$ denotes that we deliberately assign a value $q$ to variable $Q$ (i.e., intervention), rather than passively observe $Q=q$. As illustrated in Figure 3(c), $d o(Q=q)$ can be understood as cutting all the original incoming arrows to $Q$, and then making $Q$ and $U$ independent. Therefore, we can have the well-known backdoor adjustment [28]: $P(A \mid \operatorname{do}(Q=q))=\sum_{u} P(A \mid Q=$ $q, u) P(u)$. Note that this is different from Bayes rule $P(A \mid Q=q)=\sum_{u} P(A \mid Q=q, u) P(u \mid Q=q)$ thanks to the independence $P(u \mid Q=q)=P(u)$ introduced by $d o$-calculus. Let's revisit Figure 3(b) by using do-calculus. We can find that $P(A \mid d o(Q=q))-P(A)=0$, that is to say, any intervention of $Q$ will not influence the probability of $A$, meaning the correct relation between $Q$ and $A$ : no causal relation. Therefore, $P(A \mid d o(Q=q))$ should be the objective answer model in VisDial.

For our proposed graph of VisDial shown in Figure 1, we can use intervention $d o(Q, H, I)$ and the backdoor adjustment to obtain our overall model. Here, we slightly abuse the notation $d o(Q, H, I)$ as $d o(Q=q, H=h, I=i)$ :

$$
\begin{aligned}
& P(A \mid d o(Q, H, I)) \\
& =\sum_{u} P(A \mid d o(Q, H, I), u) P(u \mid d o(Q, H, I)) \\
& =\sum_{u} P(A \mid d o(Q), H, I, u) P(u \mid H) \\
& =\sum_{u} P(A \mid Q, H, I, u) P(u \mid H) .
\end{aligned}
$$


The detailed derivation and proof can be found in supplementary materials.

So far, we have provided all the ingredients of the baseline causal graph, two proposed principles and their theoretical solution: $d o$-calculus. Next, we will introduce some implementations for the proposed solution in Eq. (1).

\section{Improved Visual Dialog Models}

It is trivial to implement $\mathrm{P} 1$ and we will provide its training details in Section 6.3. For P2, since $U$ is unobserved, it is impossible to sample $u$ in Eq. (1) directly. Therefore, our technical contribution is to introduce 3 approximations. For notation simplicity, we first re-write Eq. (1) as:

$$
P(A \mid d o(Q, H, I))=\sum_{u} P_{u}(A) P(u \mid H),
$$

where $P_{u}(A):=P(A \mid Q, H, I, u)$.

\subsection{Question Type}

Since we cannot directly sample $u$ from the unobserved confounder, we use the $i$-th answer candidate $a_{i}$ as a delegate for sample $u$. That is because $a_{i}$ is a sentence observed from the "mind" of user $u$ during dataset collection. Then, $\sum_{u} P_{u}(A) P(u \mid H)$ can be approximated as $\sum_{i} P_{i}(A) P\left(a_{i} \mid H\right)$. We further use $p\left(a_{i} \mid Q T\right)$ to approximate $P\left(a_{i} \mid H\right)$ because of two reasons: First, $P\left(a_{i} \mid H\right)$ essentially describes a prior knowledge about $a_{i}$ without comprehending the whole $\{Q, H, I\}$ triplet. A similar scenario is that if we know the QT (question type), e.g. " "what color", the answer candidates denoting colors have higher probabilities without even comprehending the question details. Second, QT is extracted from question $Q$, which is a descendent of history $H$ in our graph, indicating that QT partially reveals $H$ [28]. In practice, we manually define some question types, each of which has a certain answer frequency. For each dialog round, a normalized score $s_{i}^{q t}:=p\left(a_{i} \mid Q T\right)$ (i.e., $\sum_{i} s_{i}^{q t}=1$ ) of each candidate $a_{i}$ will be calculated according to the frequency of $a_{i}$ under question type $q t$. More details are given in Section 6.3. Finally, we have the approximation for Eq. (2):

$$
\sum_{u} P_{u}(A) P(u \mid H) \approx \sum_{i} P_{i}(A) \cdot s_{i}^{q t},
$$

where $P_{i}(A)=\operatorname{softmax}\left(f_{s}\left(\boldsymbol{e}_{i}, \boldsymbol{m}\right)\right), f_{s}$ is a similarity function, $\boldsymbol{e}_{i}$ is the embedding of candidate $a_{i}, \boldsymbol{m}$ is the joint embedding for $\{Q, I, H\}$, and the sketch graph is shown in Figure 3(d). Since question type is observed from $Q$, the approximation $p\left(a_{i} \mid Q T\right)$ undermines the prior assumption of the backdoor adjustment in Eq. (1) (i.e., the prior $p(u \mid H)$ cannot be conditional on $Q$ ). Fortunately, $Q T$ is only a small part of $Q$ (i.e., the first few words) and thus the approximation is reasonable.

\subsection{Answer Score Sampling}

Since the question type implementation slightly undermines the backdoor adjustment, we will introduce a better approximation which directly samples from $u$ : Answer Score Sampling. This implementation is also widely known as our previously proposed dense fine-tune in community [3].

We still use $a_{i}$ to approximate $u$, and we use the (normalized) ground-truth NDCG score $s_{i}$ annotated by the humans to approximate $P\left(a_{i} \mid H\right)$. Note that $s_{i}$ directly reveals human preference for $a_{i}$ in the context $H$ (i.e., the prior $P\left(a_{i} \mid H\right)$ ). In practice, we use the subset of training set with dense annotations to sample $s_{i}$. Therefore, we have:

$$
\sum_{u} P_{u}(A) P(u \mid H) \approx \sum_{i} P_{i}(A) \cdot s_{i},
$$

and the sketch graph is illustrated in Figure 3(e). In practice, Eq. (4) can be implemented using different loss functions. Here we give three examples:

Weighted Softmax Loss $\left(R_{1}\right)$. We extend the log-softmax loss as a weighted form, where $P_{i}(A)$ is denoted by $\log \left(\operatorname{softmax}\left(p_{i}\right)\right), p_{i}$ denotes the logit of candidate $a_{i}$, and $s_{i}$ is corresponding normalized relevance score.

Binary Sigmoid Loss $\left(R_{2}\right)$. This loss is close to the binary cross-entropy loss, where $P_{i}(A)$ represents $\log \left(\operatorname{sigmoid}\left(p_{i}\right)\right)$ or $\log \left(\operatorname{sigmoid}\left(1-p_{i}\right)\right)$, and $s_{i}$ represents corresponding normalized relevance score.

Generalized Ranking Loss $\left(R_{3}\right)$. Note that the answer generation process can be viewed as a ranking problem. Therefore, we derive a ranking loss that $P_{i}(A)$ is $\log \frac{\exp \left(p_{i}\right)}{\exp \left(p_{i}\right)+\sum_{j \in G} \exp \left(p_{j}\right)}$, where $G$ is a group of candidates which have lower relevance scores than candidate $a_{i}$ and $s_{i}$ is normalized characteristic score (i.e., equals to 0 for $a_{i}$ with relevance score 0 and equals to 1 for $a_{i}$ with positive relevance score).

More details of the three loss functions are given in supplementary materials. It is worth noting that our losses are derived from the underlying causal principle P2 in Eq. (4), but not from the purpose of regressing to the ground-truth NDCG. The comparison will be given in Section 6.4.

\subsection{Hidden Dictionary Learning}

The aforementioned two implementations are discrete since they sample specific $a_{i}$ to approximate $u$. For better approximation, we propose learning to approximate the unobserved confounder $U$. As shown in Figure 3(f), we design a dictionary to model $U$. In practice, we design the dictionary as a $N \times d$ matrix $D_{u}$, where $N$ is manually set and $d$ is the hidden feature dimension. Note that given a sample $u$ and a answer candidate $a_{c}$, Eq.(2) can be implemented as $\sum_{u} P_{u}\left(a_{c}\right) P(u \mid H)$. Since the last layer of our network for answer prediction is a softmax layer: 
$P_{u}\left(a_{c}\right)=\operatorname{softmax}\left(f_{s}\left(\boldsymbol{e}_{\boldsymbol{c}}, \boldsymbol{u}, \boldsymbol{m}\right)\right)$, where $\boldsymbol{e}_{\boldsymbol{c}}$ is the embedding of candidate $a_{c}, \boldsymbol{u}$ is sampled from $\boldsymbol{D}_{\boldsymbol{u}}, \boldsymbol{m}$ is the joint embedding for $\{Q, I, H\}$, and $f_{s}$ is a similarity computation function, the Eq.(2) can be re-written as:

$$
P(A \mid \operatorname{do}(Q, H, I)):=\mathbb{E}_{[u \mid H]}\left[\operatorname{softmax}\left(f_{s}\left(\boldsymbol{e}_{\boldsymbol{c}}, \boldsymbol{u}, \boldsymbol{m}\right)\right)\right] .
$$

Since Eq. (5) needs expensive samplings for $\boldsymbol{u}$, we use NWGM approximation [42,35] to efficiently move the expectation into the softmax:

$\mathbb{E}_{[u \mid H]}\left[\operatorname{softmax}\left(f_{s}\left(\boldsymbol{e}_{\boldsymbol{c}}, \boldsymbol{u}, \boldsymbol{m}\right)\right)\right] \approx \operatorname{softmax}\left(\mathbb{E}_{[u \mid H]}\left[f_{s}\left(\boldsymbol{e}_{\boldsymbol{c}}, \boldsymbol{u}, \boldsymbol{m}\right)\right]\right)$,

The details of the NWGM approximation can be found in supplementary materials. In this paper, we model $f_{s}\left(\boldsymbol{e}_{\boldsymbol{c}}, \boldsymbol{u}, \boldsymbol{m}\right)=\boldsymbol{e}_{\boldsymbol{c}}^{T}(\boldsymbol{u}+\boldsymbol{m})$. Thanks to the linear additive property of expectation calculation, we can use $\boldsymbol{e}_{\boldsymbol{c}}^{T}\left(\mathbb{E}_{[u \mid H]}\left[\boldsymbol{D}_{\boldsymbol{u}}\right]+\boldsymbol{m}\right)$ to calculate $\mathbb{E}_{[u \mid H]}\left[\boldsymbol{e}_{\boldsymbol{c}}^{T}(\boldsymbol{u}+\boldsymbol{m})\right]$. In practice, we use a dot-product attention to compute $\mathbb{E}_{[u \mid H]}\left[\boldsymbol{D}_{\boldsymbol{u}}\right]$. Specifically, $\mathbb{E}_{[u \mid H]}\left[\boldsymbol{D}_{\boldsymbol{u}}\right]=\operatorname{softmax}\left(\boldsymbol{L}^{T} \boldsymbol{K}\right) \odot$ $\boldsymbol{D}_{\boldsymbol{u}}$, where $\boldsymbol{L}=\boldsymbol{W}_{\mathbf{1}} \boldsymbol{h}, \boldsymbol{K}=\boldsymbol{W}_{\mathbf{2}} \boldsymbol{D}_{\boldsymbol{u}}$ and $\odot$ is elementwise product, $\boldsymbol{h}$ is the embedding of history $H$, and $\boldsymbol{W}_{\mathbf{1}}, \boldsymbol{W}_{\mathbf{2}}$ are mapping matrices. The training details can be found in Section 6.3.

\section{Experiments}

\subsection{Experimental Setup}

Dataset. Our proposed principles are evaluated on the recently released real-world dataset VisDial v1.0. Specifically, the training set of VisDial v1.0 contains $123 \mathrm{~K}$ images from COCO dataset [20] with a 10-round dialog for each image, resulting in totally $1.2 \mathrm{M}$ dialog rounds. The validation and test sets were collected from Flickr, with $2 \mathrm{~K}$ and 8K COCO-like images respectively. The test set is further split into test-std and test-challenge splits, both with the number of $4 \mathrm{~K}$ images that are hosted on the blind online evaluation server. Each dialog in the training and validation sets has 10 rounds, while the number in test set is uniformly distributed from 1 to 10 . For each dialog, a list of 100 answer candidates is given for evaluation. In the following, the results are reported on the validation and test-std set.

Metrics. As mentioned in Section 3.1, NDCG is recommended by the official and accepted by the community. There are some other retrieval-based metrics like MRR (Mean Reciprocal Rank), where the ground-truth answer is generated by the single user. Note that the only answer may easily influenced by the single user's preference (i.e., length). We argue that this may be the reason why the models with history shortcut achieve higher MRR, (e.g., due to the bias illustrated in Figure 2) and lower NDCG. Therefore, retrieval-based metrics are not consistent with NDCG. According to the mentioned reasons and space limitation, we only show the results on NDCG in the main paper. For completeness, the further discussion between NDCG and other retrieval-based metrics and the performance on all metrics will be given in the supplementary materials.

\subsection{Model Zoo}

We report the performance of the following base models, including LF [9], HCIAE [21], CoAtt [41] and RvA [26]:

LF [9]. This naive base model has no attention module. We expand the model by adding some basic attention operations, including question-based history attention and question-history-based visual attention refinement.

HCIAE [21]. The model consists of question-based history attention and question-history-based visual attention.

CoAtt [41]. The model consists of question-based visual attention, image-question-based history attention, imagehistory-based question attention, and the final questionhistory-based visual attention.

RvA [26]. The model consists of question-based visual attention and history-based visual attention refinement.

\subsection{Implementation Details}

Pre-processing. For language pre-processing, we followed the process introduced by [9]. First, we lowercased all the letters in sentences, converted digits to words and removed contractions. After that, we used Python NLTK toolkit to tokenize sentences into word lists, followed by padding or truncating captions, questions, and answers to the length of 40, 20 and 20, respectively. Then, we built a vocabulary of the tokens with the size of 11,322, including 11,319 words that occur at least 5 times in train v1.0 and 3 instruction tokens. We loaded the pre-trained word embeddings from GloVe [30] to initialize all word embeddings, which were shared in encoder and decoder, and we applied 2layers LSTMs to encode word embedding and set their hidden state dimension to 512. For the visual feature, we used bottom-up-attention features [4] given by the official [1].

Implementation of Principles. For Principle 1 (P1), we eliminated the history feature in the final fused vector representation for all models, while kept other parts unchanged. For HCIAE [21] and CoAtt [41], we also blocked the history guidance to the image. For Principle 2 (P2), we trained the models using the preference score, which can be counted from question type or given by the official (i.e., dense annotations in VisDial v1.0 training set). Specifically, for "question type", we first defined 55 types and marked answers occurred over 5 times as preferred answers, then used the preference to train our model by $\left(R_{2}\right)$ loss proposed in Section 5.2. "Answer score sampling" was directly used to fine-tune our pre-trained model by the proposed loss functions. For "hidden dictionary", we set a matrix for $N$ as 100 and $d$ as 512 to realize $D_{u}$. The dictionary is initialized with the features of top- 100 popular answers, then trained by dense annotations with $R_{3}$ loss. More details can 


\begin{tabular}{l|c|c|cccc|c}
\hline \multirow{2}{*}{ Model } & \multirow{2}{*}{ baseline } & \multirow{2}{*}{ QT } & \multicolumn{4}{|c|}{$\mathrm{S}$} & \multirow{2}{*}{$\mathrm{D}$} \\
\cline { 4 - 7 } & & & $R_{0}$ & $R_{1}$ & $R_{2}$ & $R_{3}$ & \\
\hline LF [9] & 57.21 & 58.97 & 67.82 & 71.27 & 72.04 & 72.36 & 72.65 \\
LF +P1 & 61.88 & 62.87 & 69.47 & 72.16 & 72.85 & 73.42 & $\mathbf{7 3 . 6 3}$ \\
\hline
\end{tabular}

Table 1. Performance (NDCG\%) comparison for the experiments of applying our principles on the validation set of VisDial v1.0. $\mathrm{LF}$ is the enhanced version as we mentioned. QT, S and D denote question type, answer score sampling, and hidden dictionary learning, respectively. $R_{0}, R_{1}, R_{2}, R_{3}$ denote regressive loss, weighted softmax loss, binary sigmoid loss , and generalized ranking loss, respectively.

be found in supplementary materials. Note that the implementations following $\mathrm{P} 1$ and $\mathrm{P} 2$ are flexible.

Training. We used softmax cross-entropy loss to train the model with P1, and used Adam [17] with the learning rate of $4 \times 10^{-3}$ which decayed at epoch 5, 7, 9 with the decay rate of 0.4 . The model was trained for 15 epochs totally. In addition, Dropout [35] was applied with ratio of 0.4 for RNN and 0.25 for fully connected layers. Other settings were set by default.

\subsection{Quantitative Results}

Table 1 shows the results with different implementations in $\mathrm{P} 2$, i.e., question type, answer score sampling, and hidden dictionary learning. Overall, all of the implementations can improve the performances of base models. Specifically, the implementations of $\mathrm{P} 2$ can further boost performance by at most $11.75 \%$ via hidden dictionary learning. Speifically, our designed loss functions based on Eq. (2) outperform the regressive score, which is implemented as Euclidean distance loss and denoted as $R_{0}$. The reason is that the regression fine-tune strategy is not a proper approximation for $\mathrm{P} 2$. We also find that the proposed ranking loss (i.e., $R_{3}$ ) performs best since it satisfies the ranking property of VisDial.

Note that our principles are model-agnostic. Table 2 shows the results about applying our principles on four different models (i.e., LF [9], HCIAE [21], CoAtt [41] and RvA [26]). In general, both of our principles can improve all the models in any ablative condition (i.e., P1, P2, $\mathrm{P} 1+\mathrm{P} 2)$. Note that the effectiveness of $\mathrm{P} 1$ and $\mathrm{P} 2$ are additive, which means combining $\mathrm{P} 1$ and $\mathrm{P} 2$ performs the best.

We finally used the blind online test server to justify the effectiveness of our principles on the test-std split of VisDial v1.0. As shown in Table 3, the top part contains the results of the baseline models implemented with our principles, while the bottom part represents the recent Visual Dialog Challenge 2019 leaderboard [2]. We used the ensemble of the enhanced LF [9] to beat the Visual Dialog Challenge 2019 Winner (i.e., MReaL-BDAI), which can also be regarded as the implementations of P1 and P2. Promisingly, by applying our principles, we can promote all the baseline models to the top ranks on the leaderboard.

\begin{tabular}{l|cccc}
\hline Model & LF [9] & HCIAE [21] & CoAtt [41] & RvA [26] \\
\hline baseline & 57.21 & 56.98 & 56.46 & 56.74 \\
\hline +P1 & 61.88 & 60.12 & 60.27 & 61.02 \\
+P2 & 72.65 & 71.50 & 71.41 & 71.44 \\
+P1+P2 & $\mathbf{7 3 . 6 3}$ & 71.99 & 71.87 & 72.88 \\
\hline
\end{tabular}

Table 2. Performance (NDCG\%) of ablative studies on different models on VisDial v1.0 validation set. P2 indicates the most effective one (i.e., hidden dictionary learning) shown in Table 1. Note that only applying P2 is implemented by the implementations in Section 5 with the history shortcut.
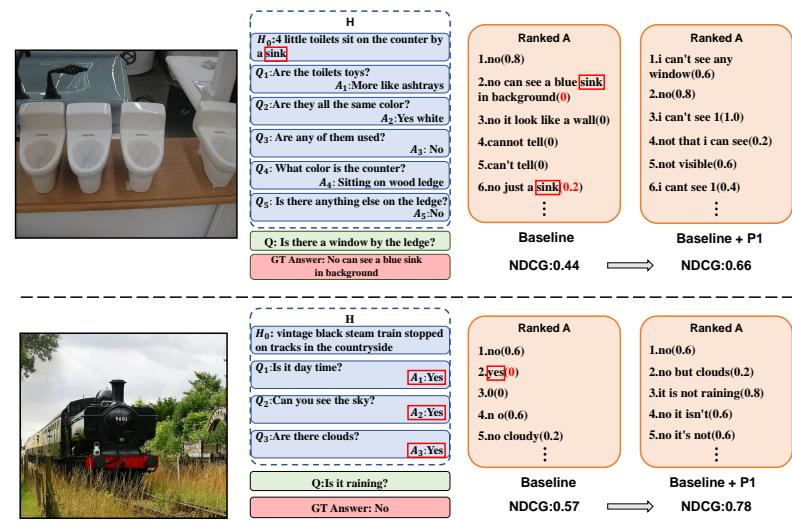

Figure 4. Qualitative results of the baseline and baseline with P1 on the validation set of VisDial v1.0. The numbers in brackets in ranked $A$ denote relevance scores. Red boxes denote that the selected candidates of the baseline model influenced by the shortcut (e.g., word matching) from the dialog history. For the baseline with P1, it does not make such biased shortcut choices. More details can be found in Section 6.5.

\subsection{Qualitative Analysis}

The qualitative results illustrated in Figure 4 and Figure 5 show the following advantages of our principles.

History Bias Elimination. After applying P1, the harmful patterns learned from history are relieved, like the answerlength bias shown in Figure 2(a) as we mentioned. The example on the top of Figure 4 shows the word-match bias in baseline. From this example, we can observe that the word "sink" from history is literally unrelated to the current question, However, for the baseline model, some undesirable candidates (i.e., with low relevance score) containing the word "sink" can be found in the top ranked answers due to the wrong direct history shortcut. To further confirm the conjecture, we counted the word-match cases of objective words (e.g., "sink" and "counter") on the validation set for the top- 10 candidates of the ranked lists. The statistic indicates that P1 can decrease about $10 \%$ word matching from history (from $\sim 5200$ times of baseline to $\sim 4800$ times using P1). The bottom example shows that, when the answer "yes" exists in history, baseline model will tend to rank "yes" in a high place. However, it is opposite to the real answer "no" in some cases, which will lead to a lower 

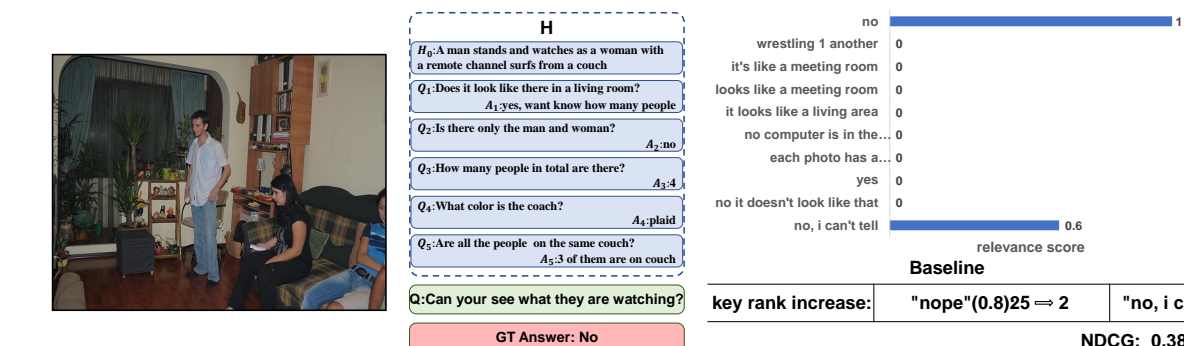
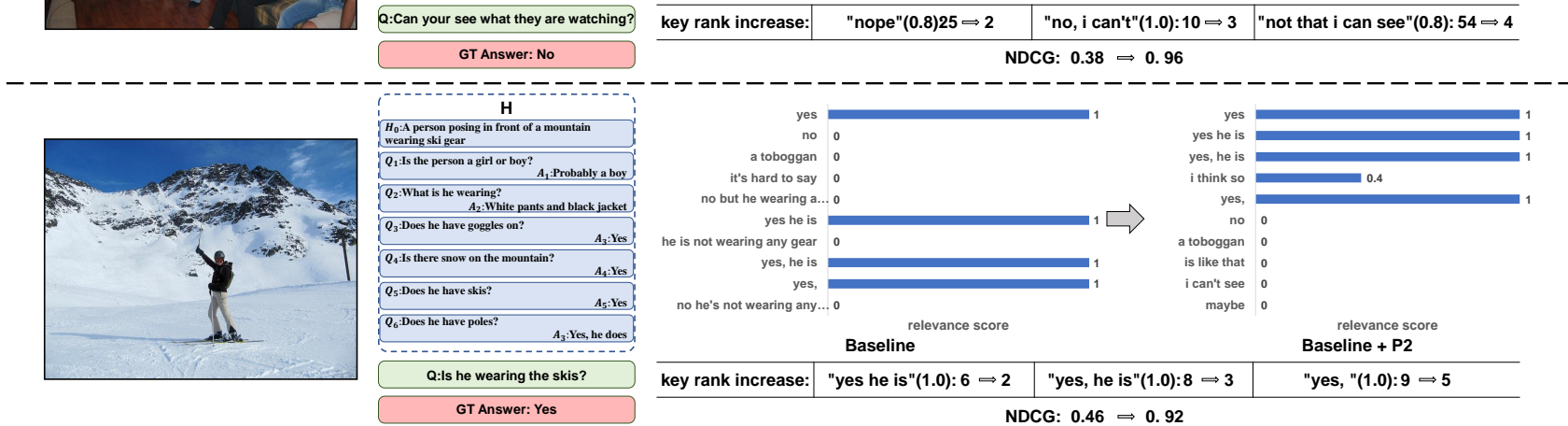

Figure 5. Qualitative examples of the ranked candidates of baseline and baseline with P2. We also give some key rank changes for boosting NDCG performance by implementing P2. These examples are taken from the validation set of VisDial v1.0.

\begin{tabular}{c|c|c}
\hline & Model & NDCG(\%) \\
\hline \multirow{5}{*}{ Ours } & P1+P2 (More Ensemble) & 74.91 \\
& LF+P1+P2 (Ensemble) & 74.19 \\
& LF+P1+P2 (single) & 71.60 \\
& RvA+P1+P2 (single) & 71.28 \\
& CoAtt+P1+P2 (single) & 69.81 \\
& HCIAE+P1+P2 (single) & 69.66 \\
\hline \multirow{5}{*}{ Leaderboard } & VD-BERT(Ensemble) & 75.13 \\
& Tohuku-CV Lab (Ensemble) $^{*}$ & 74.88 \\
& MReaL-BDAI* & 74.02 \\
& SFCU (Single) & 72.80 \\
& FancyTalk (HeteroFM) & 72.33 \\
& Tohuku-CV Lab (Ensemble w/o ft)* & 66.53 \\
\hline
\end{tabular}

Table 3. Our results and comparisons to the recent Visual Dialog Challenge 2019 Leaderboard results on the test-std set of VisDial v1.0. Results are reported by the test server, $\left({ }^{*}\right)$ denotes it is taken from [2]. Note that the top five models in the Leaderboard use the dense fine-tune implementation illustrated in Section 5.2.

NDCG. After applying P1, this problem can be effectively alleviated. To testify this conclusion, we further calculate the average ranks of "yes" for baseline and baseline with $\mathrm{P} 1$ in the above case (i.e., "yes" appears in history and real answer is "no"). We find that the average ranks are 4.82 for baseline and 6.63 for baseline with P1 respectively. The lower rank means that P1 relieves the "yes" shortcut in history. More examples of these biases can be found in supplementary materials.

More Reasonable Ranking. Figure 5 shows that the baseline model only focuses on ground truth answers like "no" or "yes" and does not care about the rank of other answers with similar meaning like "nope" or "yes, he is". This does not match human's intuition because the candidates with similar semantics are still reasonable. This also leads the baseline model to a lower NDCG. As shown in Figure 5 the model with P2 almost ranks all the suitable answers like "yes, he is", "yes he is" and "I think so" at top places together with the ground truth answer "yes", which significantly improves the NDCG performance.

\section{Conclusions}

In this paper, we proposed two causal principles for improving the VisDial task. They are model-agnostic, and thus can be applied in almost all the existing methods and bring major improvement. The principles are drawn from our in-depth causal analysis of the VisDial nature, which is however unfortunately overlooked by our community. For technical contributions, we offered some implementations on how to apply the principles into baseline models. We conducted extensive experiments on the official VisDial dataset and the online evaluation servers. Promising results demonstrate the effectiveness of the two principles. As moving forward, we will stick to our causal thinking to discover other potential causalities hidden in embodied Q\&A and conversational visual dialog tasks.

Acknowledgement This work was partially supported by National Natural Science Foundation of China (61573363 and 61832017), the Fundamental Research Funds for the Central Universities and the Research Funds of Renmin University of China (15XNLQ01), and NTU-Alibaba JRI. We would like to thank the anonymous reviewers for their constructive comments. 


\section{References}

[1] Visual Dialog. https://visualdialog.org/. 1, 3, 6

[2] Visual Dialog Challenge 2019 Leaderboard. https: //evalai.cloudcv.org/web/challenges/ challenge-page/161/leaderboard/483/.1, 7, 8

[3] Visual Dialog Challenge 2019 Winner Report. https: //drive.google.com/file/d/lfqgohregsp_ 3USM6XCHx89S9 JLXt 8bKp. 5

[4] Peter Anderson, Xiaodong He, Chris Buehler, Damien Teney, Mark Johnson, Stephen Gould, and Lei Zhang. Bottom-up and top-down attention for image captioning and visual question answering. In Proceedings of the IEEE Conference on Computer Vision and Pattern Recognition, pages 6077-6086, 2018. 2, 6

[5] Stanislaw Antol, Aishwarya Agrawal, Jiasen Lu, Margaret Mitchell, Dhruv Batra, C Lawrence Zitnick, and Devi Parikh. Vqa: Visual question answering. In Proceedings of the IEEE international conference on computer vision, pages 24252433, 2015. 1, 2

[6] Yoshua Bengio, Tristan Deleu, Nasim Rahaman, Rosemary Ke, Sébastien Lachapelle, Olexa Bilaniuk, Anirudh Goyal, and Christopher Pal. A meta-transfer objective for learning to disentangle causal mechanisms. In 8rd International Conference on Learning Representations, ICLR, 2020. 3

[7] Michael Buhrmester, Tracy Kwang, and Samuel D Gosling. Amazons mechanical turk: A new source of inexpensive, yet high-quality, data? Perspectives on Psychological Science, 6(1):3-5, 2011. 2

[8] Kyunghyun Cho, Bart van Merrienboer, Dzmitry Bahdanau, and Yoshua Bengio. On the properties of neural machine translation: Encoder-decoder approaches. In Proceedings of SSST@EMNLP 2014, Eighth Workshop on Syntax, Semantics and Structure in Statistical Translation, pages 103-111, 2014. 3

[9] Abhishek Das, Satwik Kottur, Khushi Gupta, Avi Singh, Deshraj Yadav, José MF Moura, Devi Parikh, and Dhruv Batra. Visual dialog. In Proceedings of the IEEE Conference on Computer Vision and Pattern Recognition, pages 326-335, 2017. 1, 2, 3, 6, 7

[10] Harm De Vries, Florian Strub, Sarath Chandar, Olivier Pietquin, Hugo Larochelle, and Aaron Courville. Guesswhat?! visual object discovery through multi-modal dialogue. In Proceedings of the IEEE Conference on Computer Vision and Pattern Recognition, pages 5503-5512, 2017. 2

[11] Zhe Gan, Yu Cheng, Ahmed El Kholy, Linjie Li, Jingjing Liu, and Jianfeng Gao. Multi-step reasoning via recurrent dual attention for visual dialog. In Proceedings of the 57th Conference of the Association for Computational Linguistics, ACL 2019, pages 6463-6474, 2019. 1, 2, 3

[12] Dalu Guo, Chang Xu, and Dacheng Tao. Image-questionanswer synergistic network for visual dialog. In Proceedings of the IEEE Conference on Computer Vision and Pattern Recognition, pages 10434-10443, 2019. 3

[13] Kaiming He, Xiangyu Zhang, Shaoqing Ren, and Jian Sun. Deep residual learning for image recognition. In Proceedings of the IEEE conference on computer vision and pattern recognition, pages $770-778,2016.3$
[14] Sepp Hochreiter and Jürgen Schmidhuber. Long short-term memory. Neural computation, 9(8):1735-1780, 1997. 3

[15] Unnat Jain, Svetlana Lazebnik, and Alexander G Schwing. Two can play this game: visual dialog with discriminative question generation and answering. In Proceedings of the IEEE Conference on Computer Vision and Pattern Recognition, pages 5754-5763, 2018. 2, 3

[16] Gi-Cheon Kang, Jaeseo Lim, and Byoung-Tak Zhang. Dual attention networks for visual reference resolution in visual dialog. In Proceedings of the 2019 Conference on Empirical Methods in Natural Language Processing and the 9th International Joint Conference on Natural Language Processing, EMNLP-IJCNLP, pages 2024-2033, 2019. 2, 3

[17] Diederik P. Kingma and Jimmy Ba. Adam: A method for stochastic optimization. In 3rd International Conference on Learning Representations, ICLR, 2015. 7

[18] Satwik Kottur, José MF Moura, Devi Parikh, Dhruv Batra, and Marcus Rohrbach. Visual coreference resolution in visual dialog using neural module networks. In Proceedings of the European Conference on Computer Vision (ECCV), pages 153-169, 2018. 3

[19] Ranjay Krishna, Yuke Zhu, Oliver Groth, Justin Johnson, Kenji Hata, Joshua Kravitz, Stephanie Chen, Yannis Kalantidis, Li-Jia Li, David A Shamma, et al. Visual genome: Connecting language and vision using crowdsourced dense image annotations. International Journal of Computer Vision, 123(1):32-73, 2017. 3

[20] Tsung-Yi Lin, Michael Maire, Serge Belongie, James Hays, Pietro Perona, Deva Ramanan, Piotr Dollár, and C Lawrence Zitnick. Microsoft coco: Common objects in context. In European conference on computer vision, pages 740-755. Springer, 2014. 6

[21] Jiasen Lu, Anitha Kannan, Jianwei Yang, Devi Parikh, and Dhruv Batra. Best of both worlds: Transferring knowledge from discriminative learning to a generative visual dialog model. In Advances in Neural Information Processing Systems, pages 314-324, 2017. 1, 2, 3, 6, 7

[22] Jiasen Lu, Jianwei Yang, Dhruv Batra, and Devi Parikh. Hierarchical question-image co-attention for visual question answering. In Advances In Neural Information Processing Systems, pages 289-297, 2016. 1

[23] Divyat Mahajan, Chenhao Tan, and Amit Sharma. Preserving causal constraints in counterfactual explanations for machine learning classifiers. In Advances in Neural Information Processing Systems Workshop on Causal Machine Learning, 2019. 3

[24] Suraj Nair, Yuke Zhu, Silvio Savarese, and Li Fei-Fei. Causal induction from visual observations for goal directed tasks. In Advances in Neural Information Processing Systems Workshop on Causal Machine Learning, 2019. 3

[25] Hyeonseob Nam, Jung-Woo Ha, and Jeonghee Kim. Dual attention networks for multimodal reasoning and matching. In Proceedings of the IEEE Conference on Computer Vision and Pattern Recognition, pages 299-307, 2017. 1

[26] Yulei Niu, Hanwang Zhang, Manli Zhang, Jianhong Zhang, Zhiwu Lu, and Ji-Rong Wen. Recursive visual attention in visual dialog. In Proceedings of the IEEE Conference 
on Computer Vision and Pattern Recognition, pages 66796688, 2019. 1, 2, 3, 6, 7

[27] Judea Pearl et al. Causal inference in statistics: An overview. Statistics surveys, 3:96-146, 2009. 1

[28] Judea Pearl, Madelyn Glymour, and Nicholas P Jewell. Causal inference in statistics: A primer. John Wiley \& Sons, 2016. 1, 2, 3, 4, 5

[29] Judea Pearl and Dana Mackenzie. THE BOOK OF WHY: THE NEW SCIENCE OF CAUSE AND EFFECT. Basic Books, 2018. 3, 4

[30] Jeffrey Pennington, Richard Socher, and Christopher Manning. Glove: Global vectors for word representation. In Proceedings of the 2014 conference on empirical methods in natural language processing (EMNLP), pages 1532-1543, 2014. 6

[31] Shaoqing Ren, Kaiming He, Ross Girshick, and Jian Sun. Faster r-cnn: Towards real-time object detection with region proposal networks. In Advances in neural information processing systems, pages 91-99, 2015. 3

[32] Idan Schwartz, Seunghak Yu, Tamir Hazan, and Alexander G Schwing. Factor graph attention. In Proceedings of the IEEE Conference on Computer Vision and Pattern Recognition, pages 2039-2048, 2019. 2, 3

[33] Paul Hongsuck Seo, Andreas Lehrmann, Bohyung Han, and Leonid Sigal. Visual reference resolution using attention memory for visual dialog. In Advances in neural information processing systems, pages 3719-3729, 2017. 3

[34] Rahul Singh and Liyang Sun. De-biased machine learning for compliers. In Advances in Neural Information Processing Systems Workshop on Causal Machine Learning, 2019. 3

[35] Nitish Srivastava, Geoffrey Hinton, Alex Krizhevsky, Ilya Sutskever, and Ruslan Salakhutdinov. Dropout: a simple way to prevent neural networks from overfitting. The journal of machine learning research, 15(1):1929-1958, 2014. 6, 7

[36] Kaihua Tang, Yulei Niu, Jianqiang Huang, Jiaxin Shi, and Hanwang Zhang. Unbiased scene graph generation from biased training. In Conference on Computer Vision and Pattern Recognition, 2020. 2

[37] Kaihua Tang, Hanwang Zhang, Baoyuan Wu, Wenhan Luo, and Wei Liu. Learning to compose dynamic tree structures for visual contexts. In Proceedings of the IEEE Conference on Computer Vision and Pattern Recognition, pages 66196628, 2019. 2

[38] Damien Teney, Peter Anderson, Xiaodong He, and Anton van den Hengel. Tips and tricks for visual question answering: Learnings from the 2017 challenge. In Proceedings of the IEEE Conference on Computer Vision and Pattern Recognition, pages 4223-4232, 2018. 1, 2

[39] Ashish Vaswani, Noam Shazeer, Niki Parmar, Jakob Uszkoreit, Llion Jones, Aidan N Gomez, Łukasz Kaiser, and Illia Polosukhin. Attention is all you need. In Advances in neural information processing systems, pages 5998-6008, 2017. 1

[40] Tan Wang, Jianqiang Huang, Hanwang Zhang, and Qianru Sun. Visual commonsense r-cnn. In Conference on Computer Vision and Pattern Recognition, 2020. 3

[41] Qi Wu, Peng Wang, Chunhua Shen, Ian Reid, and Anton van den Hengel. Are you talking to me? reasoned visual dialog generation through adversarial learning. In Proceedings of the IEEE Conference on Computer Vision and Pattern Recognition, pages 6106-6115, 2018. 1, 2, 3, 6, 7

[42] Kelvin Xu, Jimmy Ba, Ryan Kiros, Kyunghyun Cho, Aaron Courville, Ruslan Salakhudinov, Rich Zemel, and Yoshua Bengio. Show, attend and tell: Neural image caption generation with visual attention. In International conference on machine learning, pages 2048-2057, 2015. 3, 6

[43] Tianhao Yang, Zheng-Jun Zha, and Hanwang Zhang. Making history matter: Gold-critic sequence training for visual dialog. In Proceedings of the IEEE International Conference on Computer Vision, 2019. 2, 3

[44] Xu Yang, Kaihua Tang, Hanwang Zhang, and Jianfei Cai. Auto-encoding scene graphs for image captioning. In Proceedings of the IEEE Conference on Computer Vision and Pattern Recognition, pages 10685-10694, 2019. 2

[45] Ting Yao, Yingwei Pan, Yehao Li, and Tao Mei. Exploring visual relationship for image captioning. In Proceedings of the European Conference on Computer Vision (ECCV), pages 684-699, 2018. 2

[46] Zhou Yu, Jun Yu, Jianping Fan, and Dacheng Tao. Multimodal factorized bilinear pooling with co-attention learning for visual question answering. In Proceedings of the IEEE international conference on computer vision, pages 18211830, 2017. 1

[47] Zilong Zheng, Wenguan Wang, Siyuan Qi, and Song-Chun Zhu. Reasoning visual dialogs with structural and partial observations. In Proceedings of the IEEE Conference on Computer Vision and Pattern Recognition, pages 6669-6678, 2019. 3 


\section{Supplementary Material}

This supplementary material will give further details for the main paper, including A. More basic knowledge of causal graph for a better understanding, B. What the confounder brings to us and why we use $d o$, C. Proofs and details which are omitted in the main paper due to space limitation, D. More qualitative examples to testify the effectiveness, E. The whole tables including other metrics.

\section{A. Basic Knowledge of Causal Graph}

\section{A.1. Causal Graph}

The basic definition of causal graph is introduced in the main paper. Here, we introduce more details. The most naive configuration is $X \rightarrow Y$, which denotes $X$ causes $Y$, or $Y$ listens to $X$. This directed path from $X$ to $Y$ is called causal path, which denotes $X$ 's causal effect on $Y$. In the real world, what we want to know is the causal effect among variables, not just co-occurrence.

For easy to understand the theories we will introduce latter, we start from the simple causal graph configurations. There are three basic configurations in causal graph. 1) Chain - one arrow directed into and one arrow directed out of the middle variable-is shown in Figure 1(a). 2) Forktwo arrows emanating from the middle variable-is shown in Figure 1(b). 3) Collider - the middle variable receiving arrows from two other nodes-is like the configuration $X \rightarrow Z \leftarrow Y$, which is not shown in the picture because we will not use it.

\section{A.2. Conditional independence}

We introduce the dependency between variables in causal graph in this section. Using Chain shown in Figure 1(a) as an example, it is obvious that:

\section{$X$ and $Z$ are dependent}

i.e., for some $x, z, P(Z=z \mid X=x) \neq P(Z=z)$,

$Z$ and $Y$ are dependent

i.e., for some $z, y, P(Y=y \mid Z=z) \neq P(Y=y)$,

These two points are valid because according to the definition of causal graph, child node (i.e., $Z$ or $Y$ ) listens to its parent node (i.e., $X$ or $Z$ ).

\section{$X$ and $Y$ are likely dependent}

i.e., for some $x, y, P(Y=y \mid X=x) \neq P(Y=y)$,

$X$ and $Y$ are independent, conditional on $Z$

i.e., for all $x, y, z, P(Y=y \mid Z=z, X=x)=P(Y=y \mid Z=$ $z)$.

Here, we're only comparing cases where the value of $Z$ is constant. Since $Z$ does not change, the values of $X$ and $Y$ do not change in accordance with it. Therefore, any additional changes in the values of $X$ and $Y$ must be independent of each other. For example, we use $X, Z, Y$ to represent three events "there is fire", "there is smoke" and

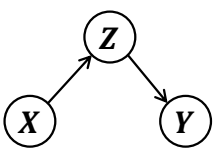

(a) Chain

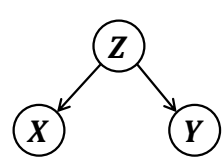

(b) Fork

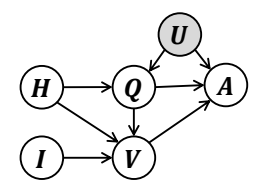

(c) Our Graph

Figure 1. Examples of some causal graph configurations and our graph of visual dialog

"smoke detector is on" respectively. If $Z$ is always equal to 1 (e.g., "there is smoke" is always true), we will find that $X$ will not influence $Y$, because whether "fire" is on, the event "smoke detector is on" is always true. Therefore, $X$ and $Y$ are independent conditional on $Z$. In the Fork shown in Figure 1(b), the conditional independence relationship among $X, Y$ and $Z$ is also satisfied (i.e., $X$ and $Y$ are independent, conditional on $Z$ ).

\section{B. Causal Effect, Confounder and $d o$}

In this section, we will give a systematical analysis of the influence of confounder, why we need $d o$ and how to calculate it. First, we need to give explanation of causal effect. Note that, in the following parts, capital letter denotes variable and lowercase denotes value.

\section{B.1. Causal Effect}

In the naive causal graph $X \rightarrow Y$, the effect of $X$ on $Y$ should be $P(Y \mid X)-P(Y)$. For the prior $P(Y)$, it is a constant. Therefore, for convenience, in this paper, we sometimes use $P(Y \mid X)$ to represent the effect of $X$ on $Y$. Note that there is only one path from $X$ to $Y$, that means the effect from $X$ on $Y$ can only pass through the causal path. So, $P(Y \mid X)$ is the causal effect. However, in the real world, things are not easy like this.

\section{B.2. Confounder}

The definition of confounder is introduced in Section 4 in the main paper. Use the Fork as an example, by the definition, we know that in Figure 1(b), $Z$ is the confounder for $X$ and $Y$. In this graph, $X$ do not have causal effect on $Y$ because if we only change $X$ and keep $Z, Y$ will not change (i.e., the causal effect of $X$ on $Y$ is 0 ). When we calculate the causal effect of $X$ on $Y$ in this graph, we find that we cannot use $P(Y \mid X)$. That is because the result of $P(Y \mid X)-P(Y)$ is not always zero as we mentioned in the Section 4.2 in the main paper. In conclusion, confounder makes us cannot use $P(Y \mid X)$ to represent the causal effect, and we need new notations to represent it.

\section{B.3. $d o$}

In the book [6], they introduce a new notation $P(Y \mid \operatorname{do}(X=x))$, which can be used to represent the 
causal effect of $X$ on $Y$. In this section, we will introduce why it can represent the causal effect and how to calculate it. Note that we will use $d o(X)$ to represent $d o(X=x)$ for concision in the following sections.

do-operator As we mentioned in the main paper, do is a type of intervention, which means that we assign a value to the variable instead of that its parent nodes cause it. For example, in Figure 1(b), $d o(X)$ is that we set variable $X$ as value $x$ ignoring its caused function (i.e., arrow $X \leftarrow Z$ ). Therefore, when we do a variable, we cut off all the arrows ending to the variable, because its parents do not cause it any more. When we calculate $P(Y \mid d o(X))$, no confounder will simultaneously cause $X$ and $Y$ because we cut of all the incoming arrows for $X$, which ensures our results are causal effect. We will give an example in Section B.4 to testify the statement.

Now, although we have a notation for causal effect, we cannot calculate it by existing methods. We need tool to derive probability formula from do formula. That is docalculus.

$\boldsymbol{d o}$-calculus Three rules of $d o$-calculus are given in [6] to help us derive probability formula.

Rule 1. When we observe a variable $X$ that is irrelevant to $Y$ (possibly conditional on other variables $Z$, like the example "Chain" in Figure 1(a)), then the probability distribution of $Y$ will not change:

$$
P(Y \mid z, X)=P(Y \mid z) .
$$

Rule 2. If a set $Z$ of variables blocks all back-door paths from $X$ to $Y$, then conditional on $Z$, like the example "Fork" in Figure 1(b), $d o(X)$ is equivalent to see $(x)$ :

$$
P(Y \mid d o(X), z)=P(Y \mid X, z) .
$$

Rule 3. We can remove $d o(X)$ from $P(Y \mid d o(X))$ in any case where there are no causal paths from $X$ to $Y$ :

$$
P(Y \mid d o(X))=P(Y) .
$$

\section{B.4. Revisit the Fork}

Now, we have $d o$-operator to represent causal effect and do-calculus to calculate it. Let us revisit the problem bring by confounder in Section B.2. In Figure 1(b), $P(Y \mid d o(X))$ can be further written as:

$$
\begin{aligned}
& P(Y \mid d o(X)) \\
& =\sum_{z} P(Y \mid d o(X), z) P(z \mid d o(X)) \\
& =\sum_{z} P(Y \mid z) P(z \mid d o(X)) \\
& =\sum_{z} P(Y \mid z) P(z) \\
& =P(Y)
\end{aligned}
$$

The first line uses Bayes rules, the second one and third one use Rule 3. As a result, $P(Y \mid d o(X))-P(Y)$ is equal to
0 , which accords with our inference for the causal effect of $X$ on $Y$ in Section B.2. That also means we can use $P(Y \mid d o(X))$ to calculate causal effect.

In conclusion, confounder makes us cannot use $P(Y \mid X)$ to represent the causal effect, and we obtain a new mathematical notation $P(Y \mid d o(X))$ to denote it. For calculating $d o$ formula, we need $d o$-calculus to derive probability formula from it, and the probability formula can be further calculated by observational data. That is the whole story of confounder and $d o$.

\section{Proofs and Details}

\section{C.1. Proofs of Equation 1}

For convenience, we draw our graph in Figure 1(c) and write down the equation again, and add one intermediate step for the formula derivation:

$$
\begin{aligned}
& P(A \mid d o(Q, H, I)) \\
& =\sum_{u} P(A \mid d o(Q, H, I), u) P(u \mid d o(Q, H, I)) \\
& =\sum_{u} P(A \mid d o(Q, H, I), u) P(u \mid d o(H)) \\
& =\sum_{u} P(A \mid d o(Q), H, I, u) P(u \mid H) \\
& =\sum_{u} P(A \mid Q, H, I, u) P(u \mid H) .
\end{aligned}
$$

According to the rules of $d o$-calculus introduced in Section B.3, we can derive the following proofs: The first step is according to the Bayes rules. The second one is due to $Q, I$ do not have a causal path to $U$ and Rule 3. Then, the third step is because $H, I$ do not have a backdoor to $A$ and Rule 2. As for the last step, although $Q$ has two backdoors to $A$ (i.e., $Q \leftarrow H \rightarrow U \rightarrow A$ and $Q \leftarrow U \rightarrow A$ ), according to Rule 2, when we control $U$, all of the backdoors are blocked. As a result, the last transformation is valid.

\section{C.2. Details of Loss Functions}

Following the Equation 4 given in Section 5.2, we give three loss functions:

Weighted $\operatorname{Softmax} \operatorname{Loss}\left(R_{1}\right)$.

$$
R_{1}=\sum_{i} \log \left(\operatorname{softmax}\left(p_{i}\right)\right) \cdot s_{i}
$$

where $p_{i}$ is the logit of candidate $a_{i}$, and $s_{i}$ is the corresponding normalized relevance score.

Binary Sigmoid Loss $\left(R_{2}\right)$.

$$
R_{2}=\sum_{i}\left[\log \left(\sigma\left(p_{i}\right)\right) \cdot s_{i}+\log \left(\sigma\left(1-p_{i}\right)\right) \cdot\left(1-s_{i}\right)\right]
$$

where $\sigma$ is the sigmoid function, $p_{i}$ is the logit of candidate $a_{i}$, and $s_{i}$ is the corresponding normalized relevance score. Generalized Ranking $\operatorname{Loss}\left(R_{3}\right)$.

$$
R_{3}=\sum_{i} \log \frac{\exp \left(p_{i}\right)}{\exp \left(p_{i}\right)+\sum_{j \in G} \exp \left(p_{j}\right)} \cdot s_{i}
$$


where $p_{i}$ is the logit of candidate $a_{i}, G$ is a group of candidates that has a lower relevance score than $a_{i} . s_{i}$ is normalized characteristic score (i.e., equals to 0 for $a_{i}$ with relevance score 0 and equals to 1 for $a_{i}$ with positive relevance score). Note that this function is reorganized from ListNet [2].

\section{C.3. Proofs of Formula 6}

By [8], we can use NWGM $\left[\operatorname{softmax}\left(f_{s}\left(\boldsymbol{e}_{\boldsymbol{c}}, \boldsymbol{u}, \boldsymbol{m}\right)\right)\right]$ (i.e., normalized weighted geometric mean) to approximate $\mathbb{E}_{[u \mid H]}\left[\operatorname{softmax}\left(f_{s}\left(\boldsymbol{e}_{\boldsymbol{c}}, \boldsymbol{u}, \boldsymbol{m}\right)\right)\right]$. If the probability of $u$ (i.e., a sample from $U$ ) is $P\left(u_{i} \mid H\right)$, and $\operatorname{softmax}\left(f_{s}\left(\boldsymbol{e}_{\boldsymbol{c}}, \boldsymbol{u}, \boldsymbol{m}\right)\right) \propto \exp \left(f_{s}\left(\boldsymbol{e}_{\boldsymbol{c}}, \boldsymbol{u}, \boldsymbol{m}\right)\right)$. We use $n_{c, i}$ to denote $f_{s}\left(\boldsymbol{e}_{\boldsymbol{c}}, \boldsymbol{u}, \boldsymbol{m}\right) . \quad \mathbb{E}_{[U \mid H]}\left[\operatorname{softmax}\left(f_{s}\left(\boldsymbol{e}_{\boldsymbol{c}}, \boldsymbol{u}, \boldsymbol{m}\right)\right)\right]$ can be written as:

$$
\begin{aligned}
& \mathbb{E}_{[u \mid H]}\left[\operatorname{softmax}\left(f_{s}\left(\boldsymbol{e}_{\boldsymbol{c}}, \boldsymbol{u}, \boldsymbol{m}\right)\right)\right] \\
& \approx \operatorname{NWGM}\left[\operatorname{softmax}\left(f_{s}\left(\boldsymbol{e}_{\boldsymbol{c}}, \boldsymbol{u}, \boldsymbol{m}\right)\right)\right] \\
& =\frac{\prod_{i} \exp \left(n_{c, i}\right)^{P\left(u_{i} \mid H\right)}}{\sum_{j} \prod_{i} \exp \left(n_{j, i}\right)^{P\left(u_{i} \mid H\right)}} \\
& =\frac{\exp \left(\mathbb{E}_{[u \mid H]}\left[n_{c, i}\right]\right)}{\sum_{j} \exp \left(\mathbb{E}_{[u \mid H]}\left[n_{j, i}\right]\right)}
\end{aligned}
$$

where $j$ is the index of all the candidates. If $f_{s}(\cdot)$ is a linear layer, the equation can be further written as:

$\mathbb{E}_{[u \mid H]}\left[\operatorname{softmax}\left(f_{s}\left(\boldsymbol{e}_{\boldsymbol{c}}, \boldsymbol{u}, \boldsymbol{m}\right)\right)\right] \approx \operatorname{softmax}\left(\mathbb{E}_{[u \mid H]}\left[f_{s}\left(\boldsymbol{e}_{\boldsymbol{c}}, \boldsymbol{u}, \boldsymbol{m}\right)\right]\right)$.

\section{C.4. Details of Principle Implementation}

Details of Enhanced LF[3]. After obtaining the vision and language feature $\mathcal{H}, \mathcal{Q}, \mathcal{I}$, we did the further operations (We use the notation Att to denote attention operation introduced in main paper): 1) History feature refine: $\tilde{\boldsymbol{h}}=\operatorname{Att}\left(\mathcal{H}, \boldsymbol{q}_{\boldsymbol{t}}\right)$, where last term of $\mathcal{Q}$ (i.e., $\boldsymbol{q}_{\boldsymbol{t}}$ ) is guidance. 2) Question and caption feature refine: $\tilde{\boldsymbol{q}}=$ $\operatorname{Att}\left(\mathcal{Q}, \boldsymbol{c}_{\boldsymbol{t}}\right), \tilde{\boldsymbol{c}}=\operatorname{Att}\left(\mathcal{C}, \boldsymbol{q}_{\boldsymbol{t}}\right)$. 3) Vision feature refine: $\tilde{\boldsymbol{v}}=\operatorname{Att}(\mathcal{V},\{\tilde{\boldsymbol{q}}, \tilde{\boldsymbol{c}}\})$. 4) Second step of vision feature refine: $\tilde{\boldsymbol{v}^{\prime}}=\operatorname{Att}\left(\tilde{\boldsymbol{v}}, \boldsymbol{g}_{v}([\tilde{\boldsymbol{h}} ; \tilde{\boldsymbol{q}}])\right)$, where $\boldsymbol{g}_{v}$ is a fully connected layer followed by a Softmax function to generate weights for refining visual attention. 5) Feature fusion: $\boldsymbol{e}=\boldsymbol{g}_{f}\left(\left[\tilde{\boldsymbol{v}^{\prime}} ; \tilde{\boldsymbol{q}}\right]\right)$, where $\boldsymbol{g}_{f}$ is a multi-head fully connected layer. More details can be found in Table 1.

Details of P2. For question type of P2, we manually defined 55 types of questions and then counted the occurrence of ground truth answers under these question types. We set answer candidates with occurrence greater than 5 as preferred answers and annotated their score as 1 under the corresponding question type. At training time, we pretrained model by original methods for 5 epochs, and gave answer candidates normalized relevance score we counted from question type for every round of each dialog. Then we used the normalized QT-relevance score to further train our

\begin{tabular}{|c|c|c|c|}
\hline Index & Input & Operation & Output \\
\hline$(1)$ & $\mathrm{H}$ (word) $($ rnd $\times 40)$ & embed and LSTM & $\mathcal{H}($ rnd $\times 512)$ \\
\hline$(2)$ & $\mathrm{C}$ (word) $(1 \times 20)$ & embed and LSTM & $\mathcal{C}(20 \times 512)$ \\
\hline$(3)$ & $\mathrm{Q}$ (word) $(1 \times 20)$ & embed and LSTM & $\mathcal{Q}(20 \times 512)$ \\
\hline$(4)$ & $\left(\mathcal{H}, q_{t}\right)$ & Attention & $h(1 \times 512)$ \\
\hline$(5)$ & $\left(\mathcal{C}, q_{t}\right)$ & Attention & $\tilde{c}(1 \times 512)$ \\
\hline$(6)$ & $\left(\mathcal{Q}, c_{t}\right)$ & Attention & $\tilde{q}(1 \times 512)$ \\
\hline$(7)$ & $(\mathcal{I}, \tilde{q}, \tilde{c})$ & Attention & $\tilde{v}(2 \times 2048)$ \\
\hline$(8)$ & $(\tilde{v},[\tilde{h} ; \tilde{q}])$ & Attention & $\tilde{v}^{\prime}(1 \times 2048)$ \\
\hline$(9)$ & $(\tilde{v}, \tilde{q})$ & Concatenate & $\boldsymbol{e}(1 \times 2560)$ \\
\hline
\end{tabular}

Table 1. The details of Enhanced LF Encoder, where rnd is the current number round of history, $\mathrm{C}$ is image caption, $\mathcal{I}$ is the image feature offered by the official with the dimension $36 \times 2048$ and $\boldsymbol{e}$ is the output of encoder.

model by $R_{2}$. For Answer Score Sampling, we pre-trained the model for 5 epochs, and then further trained the models by dense annotations with our loss functions. As for the dictionary, we set a $100 \times 512$ dictionary $D_{u}$ to explore the latent representation of $U$. We pre-trained the dictionary by one-hot ground truth answer, and then trained the dictionary by $R_{3}$ loss with the dense annotations. Then we fused the prediction of the dictionary and the prediction of pre-trained models by $\operatorname{logit}_{i}+w \cdot d_{i}$, where logit $_{i}$ is the prediction of original models, $d_{i}$ is the prediction of the dictionary, and $w$ is a manually set weight which we set as 0.1 . Finally, we further trained the whole model by $R_{3}$.

\section{C.5. Further Discussion for Metrics}

Besides NDCG, there are many other metrics like MRR (i.e., Mean Reciprocal Rank) and R@k (i.e., Recall@k). We ignore these metrics in the main paper is because we think NDCG is better than them to evaluate VisDial task. The reasons are two-fold, 1) The ground-truth for MRR and other metrics is the true answer from a user, whose answer preference (e.g., length) will be consistent in the whole 10-round dialog. Thus, if the user prefers longer answers such as "Yes, I can see a dog", then a short "Yes" will be unreasonably penalized. We argue that this may be one of the reasons why traditional models with history shortcut have higher MRR, due to the bias illustrated in Figure 2(a) in the main paper. Therefore, if a model has higher MRR, e.g.,"Yes, I can see a dog" is scored high, then it must force "Yes" to be low, leading to lower NDCG. That means other metrics (like MRR) have conflicts to NDCG. 2) As we mentioned in Section 1, the answer for VisDial is interactive but VQA has only 1 chance, thus, soft-answer score (NDCG) encourages the interaction better than 1-hot accuracy (MRR) for VisDial task. Last, Note that NDCG is recommended by VisDial organizer-A. Das-who announced in VisDial Workshop 2018 and 2019 that it is the only metric to select winners. As a result, we choose NDCG as only metric and ignore other metrics in the main paper, but for the completeness of the paper, we still give the results on other metrics in Section E. 

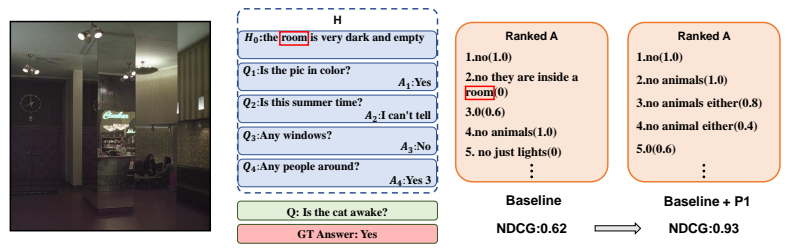

(a) Matching word "room"
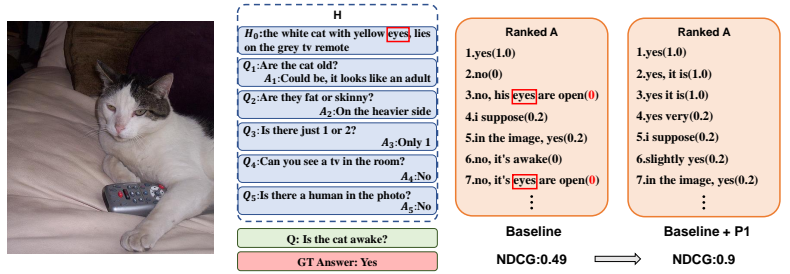

(b) Matching word "eyes"
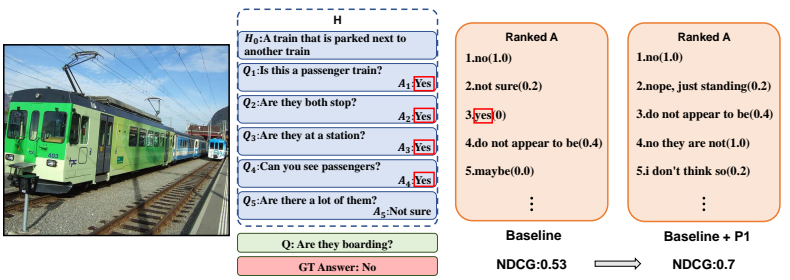

(c) Matching word "yes"
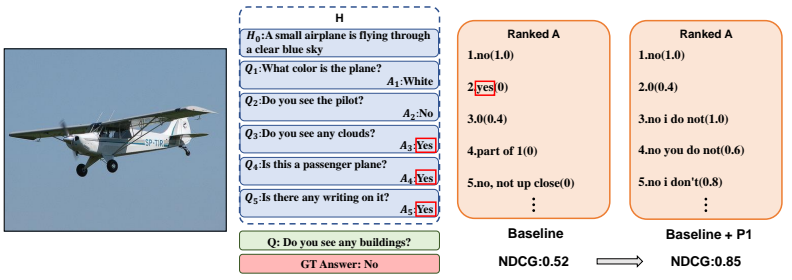

(d) Matching word "yes"

Figure 2. Word Matching

\section{More Qualitative Examples}

In this section, we will give more examples of the advantages of our principles mentioned in Section 6, including two types of history bias elimination for P1 shown in Figure 2(a) to Figure 2(d), and better ranking for P2 shown in Figure 3(a) and Figure 3(b).

\section{E. The Whole Tables}

In this section, we will give the whole tables of our experiments, especially results on other metrics omitted in the main paper. These ignored metrics are: 1) mean rank of one-hot ground truth answer (i.e., human response) (Mean), 2) recall@ $(\mathbf{R} @ \mathbf{k})$, which is the existence of the human response in the ranked top-k candidates, 3 ) mean reciprocal

\begin{tabular}{c|c|cccccc}
\hline & P2 & NDCG(\%) & MRR(\%) & R@ $1(\%)$ & R@5(\%) & R@ 10(\%) & Mean \\
\hline \multirow{6}{*}{ LF } & baseline & 57.12 & 64.33 & 50.46 & 81.41 & 90.15 & 4.03 \\
& QT & 58.97 & 64.42 & 50.70 & 81.40 & 89.93 & 4.13 \\
& $\mathrm{~S}\left(R_{0}\right)$ & 67.82 & 51.82 & 40.66 & 63.31 & 75.86 & 8.21 \\
& $\mathrm{~S}\left(R_{1}\right)$ & 71.27 & 51.40 & 38.30 & 65.54 & 78.78 & 7.09 \\
& $\mathrm{~S}\left(R_{2}\right)$ & 72.04 & 50.84 & 38.65 & 63.54 & 77.76 & 7.26 \\
& $\mathrm{~S}\left(R_{3}\right)$ & 72.36 & 50.38 & 37.13 & 64.22 & 78.09 & 7.13 \\
& $\mathrm{D}$ & 72.65 & 50.18 & 37.11 & 64.50 & 78.59 & 7.08 \\
\hline \multirow{6}{*}{$\mathrm{LF}+\mathrm{P} 1$} & baseline & 61.88 & 61.46 & 47.46 & 78.63 & 88.12 & 4.58 \\
& $\mathrm{QT}$ & 62.87 & 62.09 & 48.13 & 79.40 & 88.79 & 4.47 \\
& $\mathrm{~S}\left(R_{0}\right)$ & 69.47 & 50.54 & 39.71 & 61.41 & 74.55 & 8.72 \\
& $\mathrm{~S}\left(R_{1}\right)$ & 72.16 & 51.20 & 38.56 & 64.78 & 77.96 & 7.46 \\
& $\mathrm{~S}\left(R_{2}\right)$ & 72.85 & 50.93 & 38.88 & 63.41 & 77.66 & 7.35 \\
& $\mathrm{~S}\left(R_{3}\right)$ & 73.42 & 50.53 & 38.41 & 63.12 & 77.54 & 7.40 \\
& $\mathrm{D}$ & $\mathbf{7 3 . 6 3}$ & 50.56 & 37.99 & 63.98 & 77.95 & 7.26 \\
\hline
\end{tabular}

Table 2. The whole table of comparison for the experiments of applying our principles on the validation set of VisDial v1.0. LF is the enhanced version as we mentioned. QT, S and D denote question type, answer score sampling, and hidden dictionary learning, respectively. $R_{0}, R_{1}, R_{2}, R_{3}$ denote regressive loss, weighted softmax loss, binary sigmoid loss, and generalized ranking loss, respectively.

\begin{tabular}{c|c|cccccc}
\hline Model & P & NDCG(\%) & MRR(\%) & R@ 1(\%) & R@ 5(\%) & R@ 10(\%) & Mean \\
\hline \multirow{3}{*}{ LF [3] } & baseline & 57.12 & 64.33 & 50.46 & 81.41 & 90.15 & 4.03 \\
& $+\mathrm{P} 1$ & 61.88 & 61.46 & 47.46 & 78.63 & 88.12 & 4.58 \\
& $+\mathrm{P} 2$ & 72.65 & 50.18 & 37.11 & 64.50 & 78.59 & 7.08 \\
& $+\mathrm{P} 1+\mathrm{P} 2$ & $\mathbf{7 3 . 6 3}$ & 50.56 & 37.99 & 63.98 & 77.95 & 7.26 \\
\hline \multirow{3}{*}{ HCIAE [4] } & baseline & 56.98 & 64.13 & 50.31 & 81.42 & 90.18 & 4.09 \\
& $+\mathrm{P} 1$ & 60.12 & 61.00 & 46.66 & 78.74 & 88.34 & 4.61 \\
& $+\mathrm{P} 2$ & 71.50 & 46.96 & 32.43 & 63.47 & 78.43 & 7.28 \\
& $+\mathrm{P} 1+\mathrm{P} 2$ & 71.99 & 46.83 & 33.20 & 61.64 & 76.53 & 7.67 \\
\hline \multirow{3}{*}{ CoAtt [7] } & baseline & 56.46 & 63.81 & 49.77 & 81.20 & 90.19 & 4.13 \\
& $+\mathrm{P} 1$ & 60.27 & 60.97 & 46.83 & 78.29 & 87.86 & 4.66 \\
& $+\mathrm{P} 2$ & 71.41 & 47.32 & 33.35 & 63.51 & 77.26 & 7.56 \\
& $+\mathrm{P} 1+\mathrm{P} 2$ & 71.87 & 46.41 & 32.79 & 61.27 & 76.37 & 7.87 \\
\hline \multirow{3}{*}{ RvA [5] } & baseline & 56.74 & 64.49 & 50.67 & 81.64 & 90.50 & 3.98 \\
& $+\mathrm{P} 1$ & 61.02 & 62.00 & 47.99 & 79.14 & 89.04 & 4.42 \\
& $+\mathrm{P} 2$ & 71.44 & 50.33 & 36.85 & 64.94 & 78.81 & 7.05 \\
& $+\mathrm{P} 1+\mathrm{P} 2$ & 72.88 & 49.34 & 36.62 & 62.96 & 77.75 & 7.44 \\
\hline
\end{tabular}

Table 3. The whole table of ablative studies on different models on VisDial v1.0 validation set. P2 indicates the most effective one (i.e., hidden dictionary learning) shown in Table 2. Note that only applying P2 is implemented by the attempts in Section 5 in main paper with the history shortcut.

rank (MRR) of the human response in the returned ranked list. Note that these metrics are not suitable for visual dialog according to our discussion.

\section{References}

[1] Visual Dialog Challenge 2019 Leaderboard. https: / /evalai.cloudcv.org/web/challenges/ challenge-page/161/leaderboard/483/.

[2] Zhe Cao, Tao Qin, Tie-Yan Liu, Ming-Feng Tsai, and Hang Li. Learning to rank: from pairwise approach to listwise approach. In Proceedings of the 24th international conference on Machine learning, pages 129-136. ACM, 2007.

[3] Abhishek Das, Satwik Kottur, Khushi Gupta, Avi Singh, Deshraj Yadav, José MF Moura, Devi Parikh, and Dhruv Batra. Visual dialog. In Proceedings of the IEEE Conference on Computer Vision and Pattern Recognition, pages 326-335, 2017.

[4] Jiasen Lu, Anitha Kannan, Jianwei Yang, Devi Parikh, and Dhruv Batra. Best of both worlds: Transferring knowledge from discriminative learning to a generative visual dialog 

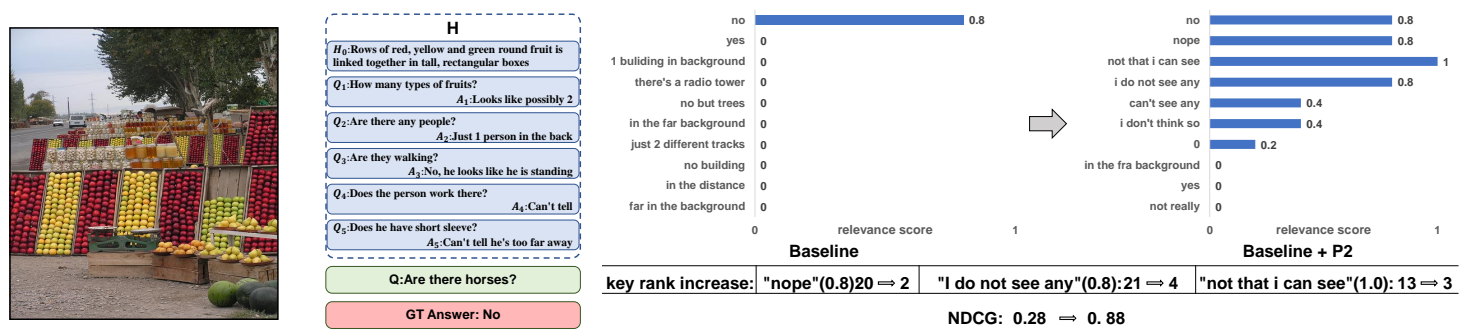

\begin{tabular}{l|l|l|l}
\hline key rank increase: "nope"(0.8)20 $\rightarrow 2$ & "I do not see any"(0.8):21 $\rightarrow 4$ & "not that $\mathrm{i}$ can see"(1.0): $13 \rightarrow 3$ \\
\hline
\end{tabular} NDCG: $0.28 \rightarrow 0.88$

(a) Better ranking for the semantics "no"
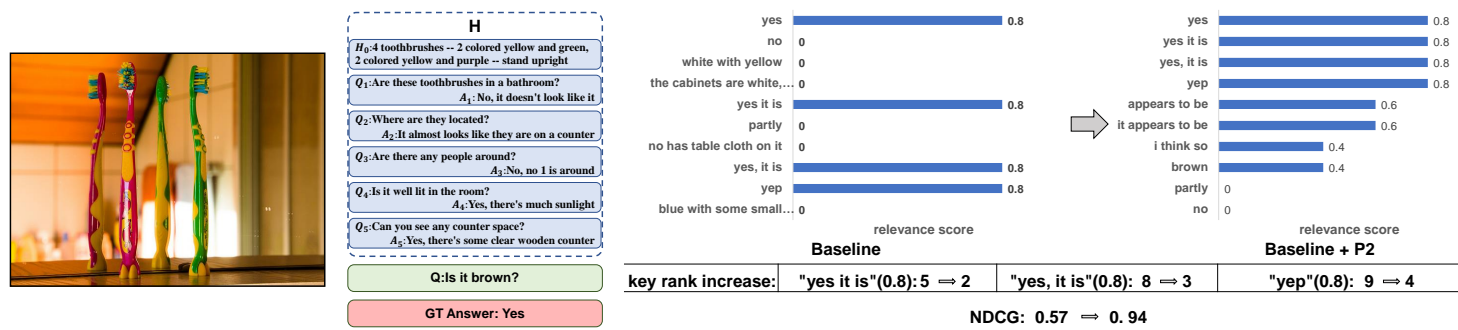

appears to be

think so 04

brown 0.4

partly 0

\begin{tabular}{l|l|l}
\hline key rank increase: & "yes it is"(0.8): $5 \Rightarrow 2$ & "yes, it is"(0.8): $8 \Rightarrow 3$
\end{tabular}

relevance scor
Baseline + P2

"yep"(0.8): $9 \Rightarrow 4$

(b) Better ranking for the semantics "yes"

Figure 3. Better Ranking

\begin{tabular}{|c|c|c|c|c|c|c|c|}
\hline & Model & $\operatorname{NDCG}(\%)$ & $\operatorname{MRR}(\%)$ & $\mathrm{R} @ 1(\%)$ & $\mathrm{R} @ 5(\%)$ & R@ 10(\%) & Mean \\
\hline \multirow{6}{*}{ Ours } & P1+P2 (More Ensemble) & 74.91 & 49.13 & 36.68 & 62.96 & 78.55 & 7.03 \\
\hline & LF+P1+P2 (Ensemble) & 74.19 & 46.69 & 32.45 & 62.13 & 77.10 & 7.33 \\
\hline & LF+P1+P2 (single) & 71.60 & 48.58 & 35.98 & 62.08 & 77.23 & 7.48 \\
\hline & $\mathrm{RvA}+\mathrm{P} 1+\mathrm{P} 2$ (single) & 71.28 & 47.71 & 34.80 & 61.53 & 77.10 & 7.63 \\
\hline & $\mathrm{CoAtt}+\mathrm{P} 1+\mathrm{P} 2$ (single) & 69.81 & 44.83 & 30.83 & 60.65 & 75.73 & 8.08 \\
\hline & $\mathrm{HCIAE}+\mathrm{P} 1+\mathrm{P} 2$ (single) & 69.66 & 44.03 & 29.85 & 59.50 & 75.98 & 8.10 \\
\hline \multirow{6}{*}{ Leaderboard } & VD-BERT(Ensemble)* & 75.13 & 50.00 & 38.28 & 60.93 & 77.28 & 6.90 \\
\hline & Tohuku-CV Lab(Ensemble)* & 74.88 & 52.14 & 38.93 & 66.60 & 80.65 & 6.53 \\
\hline & MReaL-BDAI* & 74.02 & 52.62 & 40.03 & 65.85 & 79.15 & 6.76 \\
\hline & SFCU(Single)* & 72.80 & 45.11 & 32.48 & 57.78 & 74.73 & 7.86 \\
\hline & FancyTalk(HeteroFM) ${ }^{*}$ & 72.33 & 54.56 & 42.58 & 67.27 & 80.05 & 6.37 \\
\hline & Tohuku-CV Lab(Ensemble w/o ft)* & 66.53 & 63.19 & 49.18 & 80.45 & 89.75 & 4.14 \\
\hline
\end{tabular}

Table 4. Our results and comparisons to the recent 2019 2nd Visual Dialog Challenge Leaderboard results on the test-std set of VisDial v1.0. Results are reported by the test server, $\left({ }^{*}\right)$ is taken from [1]. Note that the top five models in the Leaderboard use the dense fine-tune implementation illustrated in Section 5.1.

model. In Advances in Neural Information Processing Systems, pages 314-324, 2017.

[5] Yulei Niu, Hanwang Zhang, Manli Zhang, Jianhong Zhang, Zhiwu Lu, and Ji-Rong Wen. Recursive visual attention in visual dialog. In Proceedings of the IEEE Conference on Computer Vision and Pattern Recognition, pages 6679-6688, 2019.

[6] Judea Pearl and Dana Mackenzie. THE BOOK OF WHY: THE NEW SCIENCE OF CAUSE AND EFFECT. Basic Books, 2018.

[7] Qi Wu, Peng Wang, Chunhua Shen, Ian Reid, and Anton van den Hengel. Are you talking to me? reasoned visual dialog generation through adversarial learning. In Proceedings of the IEEE Conference on Computer Vision and Pattern Recognition, pages 6106-6115, 2018.

[8] Kelvin Xu, Jimmy Ba, Ryan Kiros, Kyunghyun Cho, Aaron Courville, Ruslan Salakhudinov, Rich Zemel, and Yoshua
Bengio. Show, attend and tell: Neural image caption generation with visual attention. In International conference on machine learning, pages 2048-2057, 2015. 